\title{
The use of thermovision technique for diagnosis of periodontal diseases and evaluation of treatment effects
}

\author{
by M. Dąbrowski ${ }^{1}$, R. Dulski ${ }^{1}$, S. Żmuda ${ }^{2}$, P. Zaborowski ${ }^{2}$ \\ (1) Military University of Technology, Institute of Optoelectronics, ul. Kaliskiego 2, \\ 00-908 Warsaw, Poland, e-mail: mirdab@wat.waw.pl \\ (2) Central Clinical Hospital of Military Medical Academy, Dentistry Institute, \\ ul. Koszykowa 78, 00-909 Warsaw, Poland
}

\begin{abstract}
The aim of this study was to create the diagnosis method of early marginal gingiva pathological states and to evaluate treatment effects.In periodontal, chronic bacterial infection is observed, which caused inflammation. Initially there are a large amount of $G$ $(+)$ cocci, then they are replaced by Actinomyces viscosus, Actinomyces israelii, Fusobacterium, Veillonella, Bacteroides and Treponeme sp. The temperature changes are often one of the earliest symptoms of pathological process. Almost all pathological conditions, for example, inflammations or cancers (tumors) caused changes of hot stream, produced in sick tissue. This process can affect temperature of surrounding tissues and often it is demonstrated on the skin and oral mucosa surfaces. Thermographic methods allow determining the value and surfaces distribution of these temperature changes.
\end{abstract}

\section{Introduction}

Oral cavity mucous membrane accomplishes a lot of important functions. It creates a covering-defence barrier and it is responsible for testing and feeling ability of absorption and secretion [1]. One can define three areas of oral mucosa with different thermal conditions appropriate to physiological conditions. The location and the blood supply of particular areas of mucosal membrane influence their thermal picture. The oral mucosa temperature distribution within gingival papillas, marginal gingiva and proper gingiva of alveolar process also depends on pathological conditions of other periodontal tissues. In pathological conditions one can observe thermal changes before clinical symptoms can be seen. The physiological difference between temperature of the same kind of tissues on both symmetrical sides of the human body was empirically fixed as $0.5^{\circ} \mathrm{C}$. Hence, it is being assumed, that any temperature change above $0.5^{\circ} \mathrm{C}$ level means the pathological process starts. For above reasons during thermovision measurements it is necessary to take into consideration physiological factors having significant influence on the thermal image of different parts of oral mucosa. These factors include location of oral mucosa area in the oral cavity and its blood supply.

The authors present investigations performed to create the diagnosis method of oral mucosa early pathological conditions with the use of the model of physiological oral mucosa temperature distribution [2].

\section{The method of investigations}

Our investigations were carried out on the group of patients aged between 40 and 50 years old. Patients were exposed under routine, preventive dental procedures. Radiological and clinical investigations using a probe and dental mirror were carried out. We eliminated pathological changes, which may have influence on thermal picture of alveolar process oral mucosa. Oral mucosa and teeth of anterior fragment of oral cavity (the areas of incisors and canines) were under investigations. Clinical changes were analysed for marginal gingiva area, gingival papillas and proper mucosal gingiva in the area of teeth injured by pathological process. A part of patients (after clinical and 
radiological investigations), who were diagnosed as having periodontium without pathological changes were used to define the model. Other patients were divided into two groups. In the first group, there were patients with acute inflammation of oral mucosa and periodontal tissue diagnosed as having bone atrophy of the second degree with teeth mobility of the first or second degree in the measuring area. In the second group, there were patients with periodontitis chronica and with bone atrophy not bigger than of the first degree with pathological pocket of 4-6 mm deep. Initial two-week treatment (procedures before surgical treatment) which included scaling, gingival pockets sterilization using $\mathrm{Nd}$ :YAG laser and biostimulation [3] was applied to the patients of this group. Another thermovision registration was carried out three days after the last dental procedure of initial treatment.

Thermographic investigation was carried out in the specified constant external conditions (air-conditioned room of constant temperature of $22^{\circ} \mathrm{C}$ ). We used the measuring thermovision camera Inframetrics ThermaCAM SC 1000 for registration of temperature distribution on the surface of particular elements of oral mucosa with the expected precision [4].

\section{The model of physiological oral mucosa temperature}

The controlled group of patients with periodontal tissues and without clinical and radiological pathological changes was prepared using standard procedures (hygienisation). The investigations were performed using thermovision camera with defined external conditions. We investigated thermal changes in the area of incisors and canines starting from oral cavity opening (in 10 seconds intervals) for 2 minutes. The measurement was repeated three times at $10 \mathrm{~min}$. intervals. Temperature of the measuring area was changing clearly within the first 30 seconds and seemed to stabilize after 60 seconds. Basing on temperature histograms of the measuring area we obtained a graph, which we called the model of physiological oral mucosa temperature distribution [2]. In order to take above two different variants of measuring conditions onto consideration we created two models. The first one reflects the temperature distribution after opening the mouth (oral cavity), and the second one the temperature distribution in stabilized conditions (after $60 \mathrm{~s}$ ).

The temperature of marginal gingiva papilla and proper oral mucosa of the alveolar process while mouth opening was between $34.2^{\circ} \mathrm{C}$ and $35.4^{\circ} \mathrm{C}$, with the average value of $34.8^{\circ} \mathrm{C}$ in the measuring area. One minute after mouth opening temperature were stabilizing at the following levels: the minimum temperature $33.3^{\circ} \mathrm{C}$, the maximum temperature $34.5^{\circ} \mathrm{C}$ and the average temperature $34.0^{\circ} \mathrm{C}$. The recorded temperature varied among particular patients' cases no more than $0.2^{\circ} \mathrm{C}$. During thermographic analyses we defined a measuring area in the way presented in Fig. 1.

We compared the temperature distributions in pathological conditions to the model.

\section{Thermographic diagnosis of periodontal diseases}

The examinations were carried out on patients with diagnosed periodontal disease by taking thermovision registration before, during and after treatment.

Thermographic registration of the marked area of mucous membrane (Fig. 1), being in physiological state, shows insignificant difference between thermograms performed at the moment of mouth opening and one minute after. During this period temperature of the investigated region decreases of about $1^{\circ} \mathrm{C}$ and slight differences of temperature are observed (Fig. 2).

For the case of acute inflammation state, significant differences in the shapes of temperature distribution of investigated areas of mucous membrane can be observed (Fig. 3). Such differences are caused by serious changes in blood supply of particular regions of mucous membrane resulting from pathological process. The comparison 
between the curves representing the moment of mouth opening and one minute after illustrates high difference in temperature of the investigated area.

After treatment of acute inflammation state, the effects of small improvement in mucous tissues can be observed both for the curves representing the moment of mouth opening and one minute after (Fig. 3). By comparing the shape of both curves with the corresponding temperature distributions representing acute conditions before treatment, one can see the decrease in temperature difference in both cases. It is caused by progressive process of tissues regeneration in particular regions of mucous membrane. By monitoring temperature distribution we can follow the treatment progress. Simultaneously, we can observe that in inflammation state it is easier to conclude on the basis of temperature distributions illustrated by the curves representing registrations made one minute after mouth opening.

For the case of chronic inflammation state of mucous membrane, also differences in temperature distributions are observed on the surface of investigated area in relation to physiological state. Thermographic image before treatment (Fig. 4) shows higher difference for the registration performed at the moment of mouth opening. One can notice the significant difference in temperature of particular regions for pathological mucous membrane with clear shift in the direction of lower temperature in relation to physiological state ones. Registration performed one minute after mouth opening is characterised by significant uniformity of temperature in the observed regions with their obvious cooling.

Thermographic registrations, made after the preliminary treatment, are the most approximate to the physiological state registration. Decision on further therapy can be made only on the basis of temperature distributions shifted in the direction of lower temperature in relation to physiological ones. The curves' shape, both at the moment of mouth opening and one minute after is very similar to physiological one. Larger shift to lower temperature, in comparison to physiological state, is observed for curve representing the moment of mouth opening.

Graphs in Fig. 5 present the thermovision evaluation of distant treatment effects. Investigations were carried out for patients with diagnosed periodontium. Treatment was efficient and periodontium tissues have been regenerated. Check-up examinations with thermographic registrations were performed after six months. Thermograms analysis confirmed positive treatment effect. The values of temperature distribution of the analysed area differed from the values of temperature distribution of physiological state of no more than $0.2^{\circ} \mathrm{C}$, i.e., they were in the range of temperature distribution assumed for model creation.

\section{Conclusions}

It results from performed investigations that thermovision technique is efficient tool for diagnosis of physiological state and pathological ones (acute and chronic) of oral cavity mucosa membrane. Both, physiological and pathological states are characterised by significant differences in temperature distribution. Examinations carried out in patients of a control group make possible, due to their repeatability, to develop a model of temperature distribution for physiological state. This model can be a reference for checking of a treatment process. Thus, thermographic analysis gives possibility of monitoring of treatment process and regeneration of periodontium tissues. Significant differences of temperature distribution in various disease entities allow for recognising them even when those changes cannot be diagnosed without invasive radiological investigations.

\section{Acknowledgement}

This research was performed under the support of the State Committee for Scientific Research of the Republic of Poland. 


\section{REFERENCES}

[1] IWANICKA-FRANKOWSKA, E., ŻMUDA, S., DYBIŻBAŃSKA, E., STRUDYCKA, I., "Decalcified freeze-dried bone allografts in periodontitis. Two years' observations", Stomatologia współczesna, nr 4/2001, p. 39-44 (in Polish)

[2] DABROWSKI, M., DULSKI, R., ŻMUDA, S., ZABOROWSKI, P., POGORZELSKI, C., "The use of thermovision camera to observe physiological and pathological conditions of oral cavity mucous membrane", Infrared Physics \& Technology, 43, 2002, p. 265-269

[3] LAURELL, L., "Treatment of intrabony defects by different surgical procedures. A literature revive", J. Periodontol, 69, 1998, p 303

[4] ASCETA, J.S., SHUMAKER, D.L., eds., "The Infrared and Electro-Optical Systems Handbook", SPIE Optical Engineering Press, Bellingham 1993
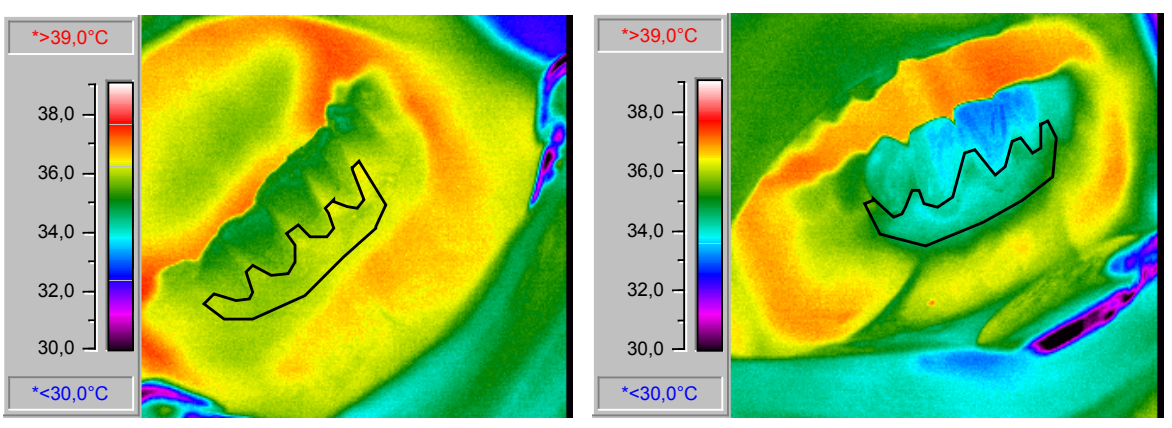

Fig. 1. Exemplary thermograms with measuring area marked (in black color)

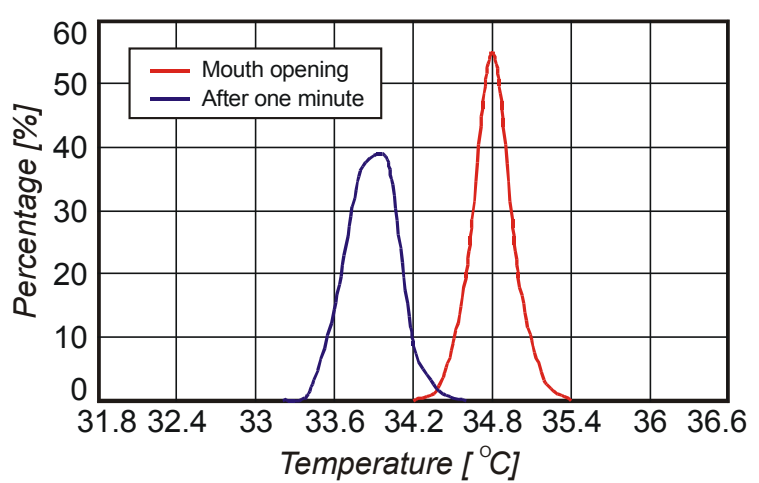

Fig. 2. The model (histogram) of physiological oral mucosa temperature distribution within measuring area 

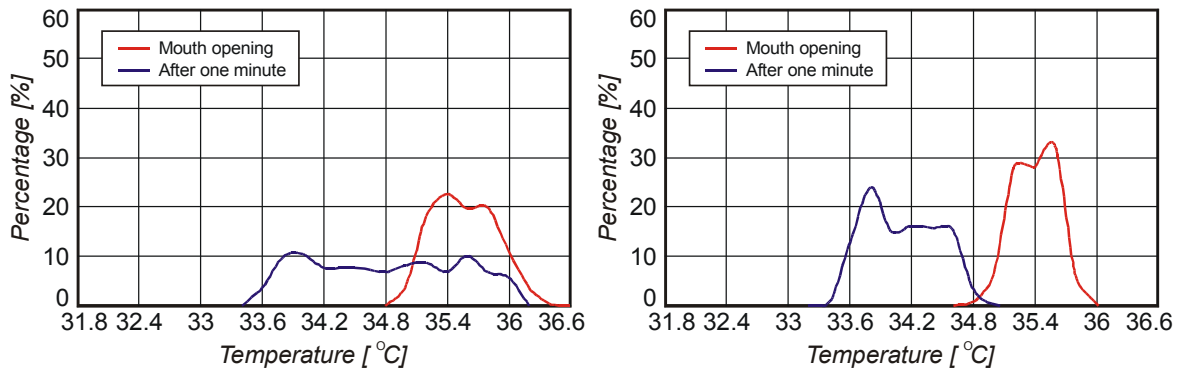

Fig. 3. Comparison between pathological acute conditions before (on the left) and after treatment (on the right)
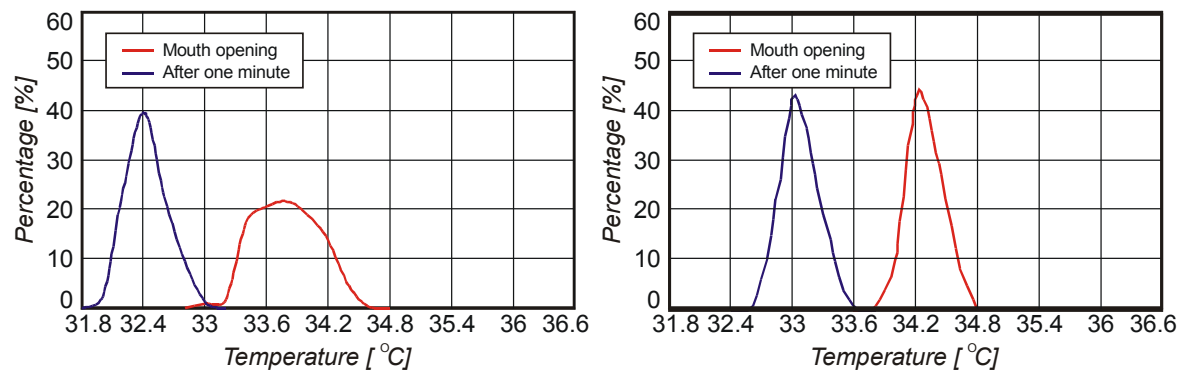

Fig. 4. Comparison between pathological chronic conditions before (on the left) and after preliminary treatment (on the right)
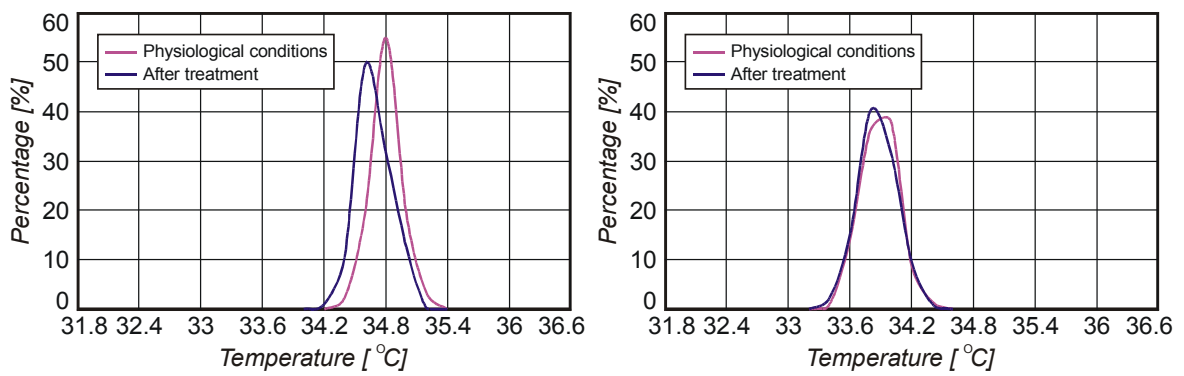

Fig. 5. Comparison of conditions after treatment and physiological one at the moment of mouth opening (on the left) and one minute after (on the right) 